DOI: http://dx.doi.org/10.20435/1025

\title{
Avaliação dos teores nutricionais de produtos comercializados em redes de fast foods no Brasil $^{1}$
}

The nutritional content evaluation of products sold in fast food chains in Brazil

\author{
Jessica Patrícia Cain ${ }^{2}$ \\ Elisvânia Freitas dos Santos ${ }^{3}$ \\ Daiana Novello ${ }^{4}$
}

${ }^{1}$ As autoras agradecem à Fundação Araucária de Apoio à Pesquisa do Estado do Paraná, pela concessão da bolsa de Iniciação Científica PIBIC/FA.

${ }^{2}$ Graduanda. Curso de Nutrição da Universidade Estadual do Centro-Oeste (UNICENTRO), Campus Cedeteg. E-mail: jessi.graff@hotmail.com

${ }^{3}$ Nutricionista, Doutora. Docente do Curso de Nutrição da Universidade Federal de Mato Grosso do Sul (UFMS). E-mail: elisvania@gmail.com

${ }^{4}$ Nutricionista, Doutora. Docente do Curso de Nutrição e do Mestrado Interdisciplinar em Desenvolvimento Comunitário da Universidade Estadual do Centro-Oeste (UNICENTRO), Campus Cedeteg. E-mail: nutridai@gmail.com 


\section{RESUMO}

Objetivou-se analisar a composição nutricional referente ao sódio, lipídios, gordura saturada e calorias informada nos rótulos de produtos comercializados em redes de fast foods do Brasil. Foram avaliados 3.120 alimentos, representados em 14 categorias de alimentos fast foods. Detectou-se moderado/ alto teor em calorias na maioria dos produtos avaliados. As pizzas e produtos de frango tiveram mais de $90 \%$ das amostras classificadas com moderado teor de gordura. Quanto ao sódio, apenas as categorias de frutas, saladas e sobremesas apresentaram produtos com baixo teor do mineral. Houve grande variabilidade nos nutrientes analisados entre empresas e produtos da mesma categoria de alimentos. Conclui-se que os produtos fast foods comercializados no país apresentam grande variabilidade de resultados e elevados teores de sódio, lipídios, gordura saturada e calorias, contribuindo para uma ingestão diária elevada. Além disso, um grande número de empresas não disponibilizam as informações nutricionais de seus produtos, violando a legislação brasileira vigente.

PALAVRAS-CHAVE

$\begin{aligned} \text { nutrientes } & \text { nutrients } \\ \text { consumidor } & \text { consumer } \\ \text { rótulo nutricional } & \text { nutrition label }\end{aligned}$

This study aimed to analyze the nutritional composition to sodium, fat, saturated fat and calories informed on product labels sold in networks of fast foods in Brazil. They were assessed 3.120 food, represented in 14 categories of fast foods. Was detected moderate/ high content of calories in most product reviews. The pizza and chicken products were more than 90\% of the samples classified with moderate fat content. As for sodium, only the categories of fruit, salads and desserts presented products with low content of mineral. There was great variability in nutrients analyzed between companies and products in the same food category. It is concluded that the fast food products marketed in the country show great variability of results and high levels of sodium, fat, saturated fat and calories, contributing to a high daily intake. In addition, a large number of companies do not provide the nutritional information of their products in violation of the current Brazilian legislation.

\section{KEY WORDS}


Avaliação dos teores nutricionais de produtos comercializados em redes de fast foods no Brasil

\section{INTRODUÇÃO}

Nas últimas décadas, houve uma mudança considerável nos hábitos alimentares da população mundial, inclusive no Brasil. Até então, a dieta era baseada em grãos frescos, tubérculos, frutas, legumes, feijão e arroz, passando-se ao crescimento constante no consumo de produtos processados e alimentação fora do domicílio, como por exemplo, os fast foods (LEAL, 2010). Entretanto o maior problema desse novo contexto alimentar é a ingestão elevada de calorias, gorduras, açúcares, sal e uma baixa ingestão de fibras, vitaminas e minerais (BRASIL, 2013). Com isso, observa-se uma transição nutricional com redução nos casos de desnutrição e aumento da obesidade e de doenças crônicas não transmissíveis como as cardiovasculares (DCV), acidente vascular cerebral (AVC), diabetes mellitus (DM) e hipertensão arterial sistêmica (HAS) (BRASIL, 2013). Segundo o estudo da Vigilância de Fatores de Risco e Proteção para Doenças Crônicas por Inquérito Telefônico (VIGITEL, 2014), no Brasil 52,5\% da população adulta está acima do peso, e 17,9\%, com obesidade. Estimativas apontam que cerca de $20 \%$ dos indivíduos adultos no país estarão com sobrepeso no ano 2025 (FRANK, 2008).

Dentre os diversos nutrientes presentes nos alimentos, destaca-se o sódio $(\mathrm{Na})$. Isso, porque a literatura científica vem demonstrando uma forte relação entre o consumo de alimentos com elevados teores de $\mathrm{Na}$ e o desenvolvimento de HAS, a qual pode elevar o risco de DCV (IBGE, 2013). No Brasil, a DCV é uma das principais causas de morte, sendo que existem, atualmente, 17 milhões de hipertensos no país. A previsão é que, até o ano de 2025, o número de hipertensos nos países em desenvolvimento deverá crescer 80\% (SBH, 2011).

No Brasil, o consumo médio estimado de sal ( $\mathrm{NaCl})$ é 10,3 g (4,11 g Na) (POWLES et al., 2013), o dobro do recomendado pela Organização Mundial de Saúde que é de $<5 \mathrm{~g}$ de $\mathrm{NaCl}(<2 \mathrm{~g}$ de 
Na) (WHO, 2013). Do total de sódio consumido, 13,8\% provém de alimentos industrializados, $6,0 \%$ do pão francês, 4,7\% de alimentos in natura e 4,1\% de alimentos semielaborados (ABIA, 2013). Porém a maior parte do sódio ingerido deriva do sal de adição e de condimentos à base de sal $(74,4 \%)$ adicionados às preparações (SARNO et al., 2013).

A população brasileira apresenta um grande consumo de fast foods, principalmente entre aqueles indivíduos pertencentes a uma classe econômica mais elevada (MORATOYA et al., 2013). Isso se deve ao fato de as pessoas estarem cada vez mais dependentes de refeições rápidas. Entretanto os fast foods são, em geral, oferecidos em grandes porções, como os hambúrgueres (95 g), por exemplo, os quais podem conter elevados teores de calorias $(250,8 \mathrm{kcal} / 100 \mathrm{~g})$, gorduras saturadas $(3,50 \mathrm{~g} / 100 \mathrm{~g})$ e $\mathrm{Na}$ (494 mg/ $100 \mathrm{~g}$ ) (USDA, 2012), colaborando para elevar o risco de doenças crônicas.

A maioria das redes de fast foods existentes no Brasil foi estabelecida primeiramente nos Estados Unidos, fato que influenciou diretamente os hábitos alimentares dos brasileiros. Dados mais recentes informam que o setor de fast food no país é constituído por 280 redes, com um total de mais de 8.600 lojas em operação (ABIA, 2011) e faturamento de food service próximo a 132,5 bilhões/ ano em 2014 (ABIA, 2014).

Apesar do grande potencial para o desenvolvimento do segmento de fast food, o cenário atual demonstra uma grande preocupação dos consumidores e organizações governamentais para um consumo de alimentos mais nutritivos, que possam reduzir os agravos à saúde (BRASIL, 2013). Nesse contexto, a indústria mundial de produtos rápidos para o consumo tem desenvolvido iniciativas voluntárias que se destinam a melhorar o perfil nutricional de seus produtos. Dentre elas citam-se a descrição de informações nutricionais nos produtos, bem como a oferta e campanhas de ingestão de alimentos mais saúdaveis, como saladas e 
Avaliação dos teores nutricionais de produtos comercializados em redes de fast foods no Brasil

frutas e preparações com ingredientes menos calóricos (DOWNS, 2013). Apesar disso, pesquisas internacionais demonstram que os nutrientes podem variar consideravelmente entre as marcas de fast foods e entre os países (DUNFORD et al., 2010). Ressaltase que, até o momento, não foram divulgadas investigações sobre os teores de nutrientes em fast foods no Brasil, apesar de alguns estudos indicarem um consumo alarmante desses produtos por todas as faixas etárias (YOKOO et al., 2013).

A informação nutricional de alimentos embalados na forma de rotulagem é bastante recente no mundo, incluindo no Brasil. Segundo a legislação brasileira, as informações nutricionais são obrigatórias apenas em rótulos de alimentos industrializados (BRASIL, 2003). Entretanto, a partir de 2010, mais de 60 restaurantes, lanchonetes e redes fast foods já estão disponibilizando informações nutricionais de seus produtos (BRASIL, 2010). Considerando esse contexto, o objetivo da pesquisa foi analisar a composição nutricional (sódio, lipídios, gordura saturada e calorias) informada nos rótulos de produtos comercializados em cadeias de fast foods do Brasil.

\section{MATERIAL E MÉTODOS}

\subsection{Coleta de dados}

Foram avaliados os conteúdos de sódio, lipídios, gordura saturada e calorias dos rótulos de alimentos fast foods comercializados no Brasil, entre agosto e outubro de 2015, similarmente ao estudo de Blonval et al. (2014). Os dados foram obtidos a partir do web site das empresas e por solicitação via atendimento ao consumidor (e-mail e telefone). Segundo a Associação Brasileia de Franchising (ABF, 2014), o Brasil apresentava, em 2014, um total de 685 redes de alimentação. Desse total, 224 foram selecionadas para a presente pesquisa por serem empresas tipo fast foods. 
Os critérios de inclusão para a seleção das empresas foram: a) possuir tabela nutricional dos produtos comercializados (web site ou e-mail); b) informar a porção do alimento em gramas e/ou por $100 \mathrm{~g}$ do alimento; c) disponibilizar todas as informações de pelo menos um de seus produtos e; c) possuir lojas em mais de três estados brasileiros. Os critérios de exclusão foram: a) apresentar lojas em menos de três estados brasileiros (112 empresas); b) não retornar a nenhuma das três tentativas de contato (42 empresas); c) estar em fase de construção e adequação das informações nutricionais (nove empresas); d) não apresentar a tabela nutricional (seis empresas); e) não fornecer as informações de tamanho de porção e/ou informações nutricionais por $100 \mathrm{~g}$ ou por porção do alimento (11 empresas). Assim, foi possível avaliar de forma completa 44 (19,64\% do total) redes de fast foods presentes no Brasil, obtendo-se um total de 3.120 alimentos.

Os produtos foram organizados em uma planilha contendo nome da empresa, nome do produto, tamanho da porção (g), calorias (kcal), lipídios (g), gordura saturada (g) e teor de sódio (mg). Esses componentes foram descritos em porção e em $100 \mathrm{~g}$ de produto. No caso de a empresa fornecer os valores nutricionais de apenas umas das formas avaliadas (porção ou 100 g), optou-se por realizar o cálculo teórico do valor faltante em função daquele fornecido.

\subsection{Definições de categorias de produtos}

Catorze categorias de fast foods foram definidas com base em instrumentos definidos pelos Estados Unidos (O' DONNELL et al., 2008) e Austrália (DUNFORD et al., 2012), bem como pela indicação de outros grupos de produtos utilizados pela indústria brasileira de fast food (DUNFORD et al., 2010; LEAL, 2010). Assim, os produtos foram classificados em: "produtos de frango" (nuggets, coxinhas, frituras, grelhados ou frango assado); "pizzas"; "produ- 
Avaliação dos teores nutricionais de produtos comercializados em redes de fast foods no Brasil

tos de batata" (purês, fritas, cozidos com ou sem condimentos); "arroz e massas"; "saladas" (todos os itens utilizados para saladas (verduras e legumes) e saladas com ingredientes adicionais (maionese e carnes); "sanduíches"; "molhos, condimentos e caldas"; "sobremesas"; "produtos a base de carnes" (carne suína, bovina e derivados); "produtos à base de frutas" (frutas, sucos de frutas e sobremesas com frutas); "porções e adicionais"; "pratos feitos"; "produtos de peixe"; e "outros" (ovo, omelete, sopa, feijoada, farofa, cebola empanada).

A categoria "outros" foi acrescida com a finalidade de computar os produtos descritos em menor número pelas empresas. A meta central para o sistema de categorização foi ser aplicável à indústria brasileira, refletindo-se, assim, os padrões de compra dos consumidores. As refeições do café da manhã foram excluídas da análise porque nem todos os restaurantes ofereciam esses menus.

\subsection{Análise dos dados}

Medidas descritivas (média e intervalo - mínimo e máximo) foram calculadas para cada tipo de alimento e nutriente presente no rótulo, tanto por porção como por $100 \mathrm{~g}$, de modo geral e para cada empresa. 0 valor diário de referência (VD) das amostras foi calculado por porção, com base na recomendação de consumo ideal de sódio de $2.000 \mathrm{mg} /$ dia (WHO, 2013), calorias $2.000 \mathrm{kcal} /$ dia (BRASIL, 2003), lipídios 100,55 g/ dia (DRI, 2005) e gordura saturada 12,3 g/ dia (DRI, 2005) para um adulto saudável (70 kg).

As seguintes categorias foram utilizadas para classificação dos nutrientes: a) sódio: baixo teor (sólido: $\leq 120 \mathrm{mg} / 100 \mathrm{~g}$; líquido: $\leq 120 \mathrm{mg} / 100 \mathrm{ml}$ ); moderado teor (sólido: > 120 a $\leq 600$ mg/ 100 g; líquido: > 120 a $\leq 300 \mathrm{mg} / 100 \mathrm{ml}$ ) e; alto teor (sólido: $>600 \mathrm{mg} / 100$ g; líquido: > $300 \mathrm{mg} / 100 \mathrm{ml}$ ) (DH, FSA, LCWG, TSG, 2013); b) lipídios: baixo teor (sólido: $\leq 3 \mathrm{~g}$ / 100 g; líquido: $\leq 1,5 \mathrm{~g} / 100 \mathrm{ml}$ ); moderado teor (sólido: > 3,0 a $\leq 17,5 \mathrm{~g} / 100 \mathrm{~g}$; 
líquido: > 1,5 a $\leq 8,75 \mathrm{~g} / 100 \mathrm{ml}$ ); alto teor (sólido: > 17,5 g /100 g; líquido: > 8,75 g/ $100 \mathrm{ml}$ (DH, FSA, LCWG, TSG, 2013); c) gordura saturada: baixo teor (sólido: $\leq 1,5 \mathrm{~g} / 100 \mathrm{~g}$; líquido: $\leq 0,75 \mathrm{~g} / 100$ $\mathrm{ml}$ ); moderado teor (sólido: > 1,5 a $\leq 5,0 \mathrm{~g} / 100$ g; líquido: > 0,75 a $\leq 2,5 \mathrm{~g} / 100 \mathrm{ml}$ ); alto teor (sólido: > 5,0 g/ 100 g; líquido: > 2,5 g/ 100 ml) (DH, FSA, LCWG, TSG, 2013) e; d) calorias: não contém ( $\leq 4 \mathrm{kcal} / 100 \mathrm{~g}$ ou ml); baixo teor ( $>4 \mathrm{a} \leq 40 \mathrm{kcal} / 100 \mathrm{~g}$ ou ml); moderado/ alto teor ( $>40 \mathrm{kcal} / 100 \mathrm{~g}$ ou ml) (BRASIL, 2012).

$\mathrm{Na}$ análise estatística, foi utilizada a análise de variância (ANOVA), one-way, para verificação da variabilidade entre os valores, sendo utilizado o teste de Tukey para comparação de médias. Os resultados foram analisados com auxílio do software Statgraphics Plus ${ }^{\circledR}$, versão 5.1, avaliados com nível de 5\% de significância.

\section{RESULTADOS}

Na Tabela 1, são apresentadas as categorias de fast foods, as quantidades de empresas e de amostras analisadas. 
Avaliação dos teores nutricionais de produtos comercializados em redes de fast foods no Brasil

Tabela 1 - Tipos, quantidades de empresas e amostras de produtos fast foods avaliadas na pesquisa, Guarapuava, PR, Brasil, 2016

\begin{tabular}{lcccc}
\hline Tipos de produtos* & \multicolumn{2}{c}{ Empresas } & \multicolumn{2}{c}{$\begin{array}{c}\text { Amostras do } \\
\text { produto }\end{array}$} \\
& $(\mathrm{n})$ & $(\%)$ & $\mathrm{C}$ (n) & $(\%)$ \\
\hline Sobremesas & 31 & 13,60 & 944 & 30,26 \\
Arroz/massas & 29 & 12,72 & 555 & 17,79 \\
Saladas & 22 & 9,65 & 119 & 3,81 \\
Molhos/condimentos/caldas & 21 & 9,21 & 165 & 5,29 \\
Sanduíches & 17 & 7,46 & 232 & 7,44 \\
Produtos de frango & 16 & 7,02 & 62 & 1,99 \\
Outros & 15 & 6,58 & 74 & 2,37 \\
Produtos de batata & 14 & 6,14 & 34 & 1,09 \\
Produtos à base de frutas & 13 & 5,70 & 421 & 13,49 \\
Porções/adicionais & 12 & 5,26 & 89 & 2,85 \\
Produtos à base de carnes & 11 & 4,82 & 81 & 2,60 \\
Produtos de peixe & 11 & 4,82 & 174 & 5,58 \\
Pratos feitos & 10 & 4,39 & 57 & 1,82 \\
Pizzas & 6 & 2,63 & 113 & 3,62 \\
Total & 228 & 100 & 3.120 & 100 \\
\hline
\end{tabular}

*ordem decrescente de número de empresas que comercializavam o produto.

Das categorias de fast foods, as sobremesas tiveram o maior percentual de comercialização, bem como maior quantidade de amostras oferecidas entre as empresas analisadas. Contudo a categoria de pizzas foi encontrada em um menor número das redes. Destaca-se que o número de produtos oferecidos pelas empresas variou de 34 (produtos de batata) a 944 (sobremesas). Segundo a Associação Brasileira de Nutrologia (ABRAN, 2010), as sobremesas são muito consumidas no Brasil, sendo que cerca de $70 \%$ da população ingere doces após as principais refeições. Também se destaca que, muitas vezes, a refeição oferecida em fast foods vem acompanhada de brindes ou sobremesas, fato que pode aumentar ainda mais o seu consumo (SANTOS; OLIVEIRA, 2014).

Na Tabela 2, são apresentados os teores de sódio, energia, lipídios e gorduras saturadas presentes em rótulos nutricionais de alimentos fastfoods e o VD por porção média de consumo diário. 
Jessica P. CAIN; Elisvânia F. dos SANTOS; Daiana NOVELLO 
Avaliação dos teores nutricionais de produtos comercializados em redes de fast foods no Brasil

Considerando-se os resultados de energia, maiores teores (100 g) foram verificados para as porções e adicionais, seguidos de arroz e massas e sobremesas. Os menores resultados foram constatados para os produtos à base de frutas seguidas por saladas. Já na porção do produto, o prato feito teve o maior nível de energia seguido pelos sanduíches, enquanto menores conteúdos foram observados para as porções e adicionais, seguidas por molhos, condimentos e caldas.

Em relação aos lipídios, maiores teores (100 g) foram observados para as porções e adicionais e sanduíches, seguido por carnes, produtos de batata e sobremesas. Porém menores conteúdos de lipídios foram observados nos produtos à base de frutas, seguidas pelas saladas. Na porção, maiores teores foram constatados para sanduíches, carnes e prato feito, enquanto menores quantidades foram verificadas para os produtos à base de frutas e porções e adicionais, seguidas por molhos, condimentos e caldas e sobremesas.

As gorduras saturadas tiveram maior teor médio (100 g) na categoria de pizzas seguida por porções e adicionais. Já, menores conteúdos foram observados para os produtos à base de frutas, seguidas por saladas. Na porção, observou-se maiores quantidades de gorduras saturadas nas pizzas, seguidas pelas carnes, prato feito e sanduíches. Os menores teores foram observados em produtos à base de frutas e porções e adicionais, seguidas por molhos, condimentos e caldas, produtos de peixe e saladas.

Na avaliação de sódio (100 g), constatou-se que os molhos, condimentos e caldas tiveram os maiores teores, seguidos pelas porções e adicionais. Menores quantidades de sódio foram verificados para os produtos à base de frutas seguidos das sobremesas. Por porção, a categoria de prato feito apresentou a maior quantidade de sódio, seguida pelos sanduíches, enquanto menores valores foram constatados para as porções e adicionais, seguidas de produtos à base de frutas e sobremesas. 
Avaliando-se a contribuição das categorias de produtos para o VD (porção média) destacam-se os seguintes resultados: a) energia - o prato feito e os sanduíches tiveram uma contribuição acima de $25 \%$, enquanto produtos à base de frutas, molhos, condimentos e caldas, porções e adicionais, saladas e sobremesas tiveram contribuições $\leq$ a $10 \%$ do consumo diário; b) lipídios - as carnes, prato feito e sanduíches apresentaram maiores porcentagens para o VD, com destaque para os sanduíches (41,85\%). De forma similar ao teor calórico, produtos à base de frutas, molhos, condimentos e caldas, porções e adicionais e saladas e sobremesas tiveram contribuições $\leq$ a 10\% do VD; c) gorduras saturadas - a maioria dos produtos apresentaram valores $\geq$ a $25 \%$ do VD (arroz e massas, carnes, pizzas, prato feito, produtos de batata, produtos de frango, sanduíches, sobremesas e outros), com destaque para as pizzas $(117,03 \%)$, carnes $(75,41 \%)$, sanduíches $(86,83 \%)$ e prato feito $(73,24 \%)$ que tiveram valores bem elevados. Nenhum produto apresentou VD $\leq 10 \%$ para esta avaliação; d) sódio - a maioria dos produtos apresentaram valores $\geq$ a $25 \%$ do VD (arroz e massas, carnes, pizzas, prato feito, produtos de frango, sanduíches e outros), ressaltando-se o prato feito $(75,44 \%)$ e sanduíches $(53,39 \%)$, com valores superiores a $50 \%$. Apenas os produtos à base de frutas, porções e adicionais e sobremesas apresentaram $\mathrm{VD} \leq 10 \%$ neste nutriente.

Na Figura 1, verifica-se a distribuição percentual de calorias, lipídios, gorduras saturadas e sódio, conforme a quantidade presente em $100 \mathrm{~g}$ dos produtos avaliados. 
Avaliação dos teores nutricionais de produtos comercializados em redes de fast foods no Brasil 
A maioria dos produtos avaliados em todas as categorias apresentaram moderado/ alto teor em calorias (variação entre 69,2 a 100\%), sendo que, em 7 grupos (50\%), foram verificados alimentos contendo baixo teor/ não contém calorias (variação entre 0,18 a $30,64 \%$ ).

Menores teores de lipídios (baixo teor) foram constatados nos grupos dos produtos à base de frutas $(81,24 \%)$, molhos, condimentos e caldas $(37,58 \%)$ e saladas $(55,46 \%)$. As demais categorias apresentaram mais que $50 \%$ de seus produtos com moderado/ alto teor de lipídios. Destacam-se as pizzas e produtos de frango, que tiveram mais que $90 \%$ das amostras consideradas com moderado teor de gordura.

$\mathrm{Na}$ avaliação da gordura saturada, maiores teores (moderado/ alto) foram observados nas categorias de arroz e massas $(37,3 \%)$, produtos à base de carnes $(51,85 \%)$, pizzas $(72,57 \%)$, porções e adicionais $(52,81 \%)$, produtos de batata $(47,06 \%)$, sanduíches $(53,45 \%)$, sobremesas $(40,15 \%)$ e outros (50\%). Ressaltase que os produtos à base de frutas, molhos, condimentos e caldas, pratos feitos, produtos de peixe e saladas mostraram mais que $50 \%$ dos alimentos contendo baixo teor de gorduras saturadas.

Quanto ao sódio, apenas as categorias de frutas (99,52\%), saladas $(45,38 \%)$ e sobremesas $(89,51 \%)$ apresentaram a maioria dos produtos com baixo teor do mineral. Já os grupos de arroz e massas (64,5\%), produtos à base de carnes (51,85\%), pizzas $(73,45 \%)$, prato feito $(71,93 \%)$, produtos de batata $(70,59 \%)$, produtos de frango $(62,9 \%)$, produtos de peixe $(59,77 \%)$ e sanduíches $(80,17 \%)$ foram avaliados com mais de $50 \%$ dos produtos contendo moderado teor de sódio.

\section{DISCUSSÃO}

A variação entre os conteúdos de energia, lipídios, gorduras saturadas e sódio entre empresas e produtos da mesma categoria 
Avaliação dos teores nutricionais de produtos comercializados em redes de fast foods no Brasil

de alimentos fast foods comercializados no Brasil foi bastante considerável. Esse fato demonstra a necessidade de novos investimentos que visem reduzir e/ ou substituir os ingredientes com elevados teores de nutrientes classificados como prejudiciais à saúde (DUNFORD et al., 2010; 2012).

Alguns exemplos merecem destaque quanto à variação nos teores de nutrientes (100 g) entre os mesmos produtos e marcas (Tabela 2). É o caso de produtos como arroz e massas e molhos, condimentos e caldas que apresentaram aproximadamente 119 e 3.846; 2.353 e 12.000 vezes de variação nos teores de energia e sódio, respectivamente. Também, as porções adicionais e produtos de peixe com variabilidade de 2.793 e 8.200 vezes, respectivamente, no conteúdo de sódio. Esses resultados estão de acordo com outras pesquisas realizadas na Austrália, EUA, Reino Unido e Costa Rica (DUNFORD et al., 2012; BLONVAL et al., 2014).

Produtos como prato feito e sanduíches apresentaram teores acima de 25\% de contribuição do VD diários em energia, lipídios, gorduras saturadas e sódio. Já o arroz e massas, produtos à base de carne, pizzas, prato feito, produtos de frango, sanduíches e outros tiveram contribuição do VD diário acima de 25\% em gorduras saturadas e sódio, sendo aqueles com maior destaque na presente pesquisa. Efeitos similares foram relatados por Rangan et al. (2009) avaliando produtos fast foods (batatas fritas, pizzas e refrigerantes) comercializados na Austrália. Os resultados observados são considerados alarmantes, uma vez que um consumo elevado e/ ou frequente desses alimentos pode aumentar o risco de doenças como obesidade, DM, HAS e dislipidemias, dentre outras (WHO, 2003).

Cabe ressaltar que produtos como molhos, condimentos e caldas e porções adicionais, quando avaliados apenas pela porção, apresentaram baixos teores de energia, lipídios, gorduras saturadas e sódio. Contudo deve-se lembrar que, muitas vezes, esses alimentos são consumidos diariamente e várias vezes ao dia, o 
que também aumenta as chances para a ocorrência de patologias crônicas (SILVA et al., 2015).

Os resultados da presente pesquisa são de grande relevância para a saúde pública. Nesse contexto, servem de base para monitorar a qualidade e a quantidade de calorias, sódio, lipídios e gordura saturada em produtos fast foods ao longo do tempo. Além disso, podem fornecer um ponto de partida para se definir metas e ações futuras, com intuito de reduzir em potencial esses nutrientes nos produtos comercializados, fato que já ocorre em outros países (GARCIA et al., 2014). Ademais, esses achados podem colaborar para a elaboração de novas leis e fiscalização das redes de fastfoods por órgãos públicos. Nesse caso, é fundamental que as empresas forneçam informações nutricionais claras, completas e fidedignas de seus produtos. Isso, porque pode existir uma grande variação na composição química de alimentos, dependendo da região em que foi produzido, tipo de cultivar, forma de plantio e fatores climáticos, dentre outros (CHITARRA; CHITARRA, 1990).

É importante enfatizar que um número considerável de empresas não disponibilizam ou fornecem informações nutricionais incompletas dos alimentos, seja no rótulo, no web site da empresa e/ ou por telefone. Esse fato prejudica diretamente o consumidor final, uma vez que estse público busca cada vez mais obter informações dos produtos que adquirem (VENTURA, 2010). Além disso, conforme a legislação brasileira, é direito dos consumidores receber informações fidedignas sobre a composição dos alimentos que consomem, a fim de orientar escolhas mais adequadas e saudáveis (BRASIL, 2010).

Segundo Silva et al. (2015), a falta de informação, principalmente aquela relacionada à alimentação e nutrição, pode levar o indivíduo a um consumo excessivo de alimentos considerados prejudiciais, como os fast foods. Nesse caso, pode-se elevar o risco do desenvolvimento de doenças crônicas não transmissíveis ou agravar casos em que essas doenças já estejam instaladas. 
Avaliação dos teores nutricionais de produtos comercializados em redes de fast foods no Brasil

No Brasil, a rotulagem nutricional é regulamentada pela Agência Nacional de Vigilância Sanitária (Anvisa), por meio da RDC no 360 de 23 de dezembro de 2003 (BRASIL, 2003), tornando-a obrigatória nos produtos comercializados no país. Nesse contexto, desde 2010, mais de 60 restaurantes e lanchonetes já disponibilizavam as informações nutricionais de seus produtos. Esse efeito foi motivado, principalmente, devido a um acordo realizado entre a Anvisa, o Ministério Público Federal e a Associação Nacional de Restaurantes (BRASIL, 2010). 0 intuito foi implementar a tabela nutricional em produtos e divulgar a informação nutricional por meio da embalagem, quadros, cartazes, cardápios, folders, dentre outras. Com isso, espera-se que o consumidor opte por uma refeição mais saudável quando estiver fora de casa. Além disso, entre os anos de 2007 e 2011, foram firmados termos de compromisso entre o Ministério da Saúde, Anvisa e associações representativas do setor produtivo, visando reduzir a quantidade de sal, açúcares e gorduras em produtos alimentícios processados no Brasil (BRASIL, 2010).

No caso específico do sódio, muitas são as dificuldades relatadas pelas empresas para que ocorra a redução do mineral em produtos alimentícios. Dentre elas, estão o aumento nos custos de produção e, principalmente, a alteração das propriedades sensoriais e tecnológicas dos produtos, reduzindo-se o gosto salgado e o sabor global, textura, cor, consistência e shelf life dos alimentos (NUTRIONIX, 2015). Contudo a literatura já demonstra que é possível uma redução média de $20 \%$ de gordura e $50 \%$ nos níveis de sal em fast foods como ocorre, por exemplo, na comercialização de pastel de carne na Irlanda e Reino Unido (TOBIN et al., 2012).

Diante da possibilidade de um consumo alimentar mais saudável, algumas indústrias e restaurantes de países como Chile, Argentina, México e Brasil resolveram investir nesse setor, o qual vem demonstrando ser um novo nicho de mercado (VENTURA, 2010; BLONVAL et al., 2014). Assim, é fundamental que novos 
programas e investimentos sejam realizados em nível mundial e nacional. 0 intuito deve ser direcionado a incentivar e regulamentar medidas relacionadas a uma alimentação mais saudável para que, futuramente, ocorra uma redução de doenças crônicas não transmissíveis entre a população.

\section{CONCLUSÃO}

Foi constatada uma grande variabilidade de valores de sódio, lipídios, gorduras totais e calorias entre empresas e produtos da mesma categoria de alimentos, contribuindo para uma elevada ingestão diária desses nutrientes pela população.

Em geral, os alimentos fastfoods que apresentam maiores teores de energia, lipídios, gorduras saturadas e sódio são as porções e adicionais, arroz e massas, sobremesas, prato feito, produtos de batata, carnes, molhos e condimentos, pizzas, caldas e sanduíches. Além disso, a maioria dos produtos avaliados contém moderado/ alto teor em calorias, lipídios, gordura saturada e sódio, muitas vezes ultrapassando na porção mais que a metade do valor diário de referência para o consumo.

As frutas e saladas são as categorias com menores conteúdos de energia, lipídios, gorduras saturadas e sódio e, portanto, mais benéficas à saúde.

Um elevado número de empresas não disponibilizam informações nutricionais de seus produtos, contrariando a legislação brasileira vigente. Assim, recomendam-se medidas governamentais para que haja transparência dessas informações uma vez que é um direito do consumidor saber o que está ingerindo. Também se indica um monitoramento e avaliação periódica dos produtos comercializados, para que novas metas para redução de calorias, lipídios, gorduras saturadas e sódio sejam aderidas pelas redes de fast foods brasileiras. 
Avaliação dos teores nutricionais de produtos comercializados em redes de fast foods no Brasil

\section{REFERÊNCIAS}

ASSOCIAÇÃO BRASILEIRA DAS INDÚSTRIAS DA ALIMENTAÇÃO (ABIA). Cenário do consumo de sódio pelo Brasil. Estudo elaborado com base em dados do Instituto Brasileiro de Geografia e Estatística (IBGE). São Paulo: ABIA, 2013.

. Debate sobre tendências e inovação no 8 o Congresso Internacional de Food Service. 2014. Disponível em:< http://abia.org.br/vsN/tmp_2. aspx?id=49 $\geq$. Acesso em: 20 maio 2016.

. O mercado de food service no Brasil. 2011. Disponível em: <http:// www.abia.org.br/vsn/ $\geq$ Acesso em: 3 set. 2015.

ASSOCIAÇÃO BRASILEIRA DE FRANCHISING (ABF). Inteligência de mercado. 2014. Disponível em: <http://www.abf.com.br/ $\geq$. Acesso em: 20 jan. 2016.

ASSOCIAÇÃO BRASILEIRA DE NUTROLOGIA (ABRAN). Sobremesas ao gosto dos brasileiros. 2010. Disponível em: <http://www. sebraemercados.com.br/sobremesas-ao-gosto-dos-brasileiros/ $\geq$. Acesso em: 15 abr. 2016.

BLONVAL, K. H.; METZLER, A. B.; CAMPOS, M. M.; DUNORD, E. K. The salt content of products from popular fast-food chains in Costa Rica. Appetite, v. 83, n. 1, p. 173-177, dez. 2014.

BRASIL. Agência Nacional de Vigilância Sanitária - ANVISA. Informação nutricional estará disponível em 60 redes de lanchonetes e restaurantes. 2010. Disponível em: <http://s.anvisa.gov.br/wps/s/r/e6e $\geq$. Acesso em: 20 jan. 2016.

Agência Nacional de Vigilância Sanitária - ANVISA. Resolução $R D C n^{\circ} 360$, de 23 de dezembro de 2003. Regulamento Técnico sobre Rotulagem Nutricional de Alimentos Embalados, tornando obrigatória a rotulagem nutricional. Diário Oficial da União; Poder executivo. Brasília, DF, 2003.

. Agência Nacional de Vigilância Sanitária - ANVISA. Resolução da Diretoria Colegiada - RDC No 54, de 12 de novembro de 2012. Regulamento técnico sobre informação nutricional complementar. Diário Oficial União; Poder Executivo. Brasília, DF, 2012. 
. Ministério da Saúde. Secretaria de Atenção à Saúde. Departamento de Atenção Básica. Política Nacional de Alimentação e Nutrição. Brasília: Ministério da Saúde, 2013.

CHITARRA, M. I. F.; CHITARRA, A. B. Pós-colheita de frutos e hortaliças: fisiologia e manejo. Lavras, MG: Esal, Faepe, 1990.

DEPARTMENT OF HEASLTH (DH). Food Standards Agency - FSA. Llywodraeth Cymru Welsh Governmente - LCWG. The Scottish Government - TSG. Guide to creating a front of pack (FoP) nutrition label for pre-packed products sold through retail outlets. Scotland: DH, FSA, 2013.

DIETARY REFERENCE INTAKES (DRI). Dietary Reference Intakes for energy, carbohydrate, fiber, fat, fatty acids, cholesterol, protein and amino acids. Washington: National Academy Press, 2005.

DOWNS, J. S. Does "Healthy" Fast Food Exist? The Gap Between Perceptions and Behavior. Journal of Adolescent Health, v. 53, n. 4, p. 429-430, out. 2013.

DUNFORD, E.; WEBSTER, J.; BARZI, F.; NEAL, B. Nutrient content of products served by leading Australian fast food chains. Appetite, v. 55, n. 3, p. 484-489, dez. 2010.

DUNFORD, E.; WEBSTER, J.; WOODWARD, M.; CZERNICHOW, S.; YUAN, W.; JENNER, K.; MHURCHU, C. N.; JACOBSON, M.; CAMPBELL, N.; NEAL, $B$. The variability of reported salt levels in fast foods across six countries. Opportunities for salt reduction. Canadian Medical Association Journal, v. 184, n. 9, p. 2023-2028, 2012.

FRANK, H. F. B. Obesity epidemiology. New York: Oxford University Press, 2008.

GARCIA, J.; DUNFORD, E.; SUNDSTROM, J.; NEAL, B. Changes in the sodium content of leading Australian fast-food products between 2009 and 2012. Medical Journal of Australia, v. 200, n .6, p. 340-344, 2014.

INSTITUTO BRASILEIRO DE GEOGRAFIA E ESTATÍSTICA (IBGE). Pesquisa Nacional de Saúde 2013. Percepção do estado de saúde, estilos de vida e doenças crônicas. Disponível em:< http://biblioteca.ibge.gov. br/visualizacao/livros/liv91110.pdf>. Acesso em: 2 maio 2016. 
Avaliação dos teores nutricionais de produtos comercializados em redes de fast foods no Brasil

LEAL, D. Crescimento da alimentação fora do domicílio. Segurança Alimentar e Nutricional, v. 17, n. 1, p. 123-132, 2010.

MORATOYA, E. E.; CARVALHAESG, C.; WANDER, A. E.; ALMEIDA, L. M. M. C. Mudanças no padrão de consumo alimentar no Brasil e no mundo. Revista de Política Agrícola, v. 22, n. 1, p. 72-84, 2013.

NUTRIONIX. A diferença entre redução e substituição de sódio. Food Ingredients Brasil, São Paulo, v. 17, n. 35, p. 80-83. set./nov. 2015.

O’DONNELL, S.; HOERR, S.; MENDOZA, J.; GOH, T. E. Nutrient quality of fast food kids meals. American Journal of Clinical Nutrition, v. 88, n. 5, p. 1388-1395, nov. 2008.

POWLES, J.; FAHIMI, S.; MICHA, R.; KHATIBZADEH, S.; SHI, P.; EZZATI, M.; ENGELL, R.E.; LIM, S.S.; DANAEI, G.; MOZAFFARIAN, D. Global, regional and national sodium intakes in 1990 and 2010: a systematic analysis of $24 \mathrm{~h}$ urinary sodium excretion and dietary surveys worldwide. BMJ Open, v. 23, n. 12, p.1-18, dez. 2013.

RANGAN, A. M.; SCHINDELER, S.; HECTOR, D. J.; GILL, T. P.; WEBB, K. L. Consumption of 'extra' foods by Australian adults: Types, quantities and contribution to energy and nutrient intakes. European Journal of Clinical Nutrition, v. 63, n. 7, p. 865-871, out. 2009.

SANTOS, R. F. S.; OLIVEIRA, S. C. Fast Food: um reflexo da padronização do consumo consequente da globalização das ofertas de mercado. In: CONGRESSO DE CIÊNCIAS DA COMUNICAÇÃO NA REGIÃO CENTROOESTE, 16., 8-10 de maio 2014, Águas Claras, DF. Anais... Águas Claras, DF: UCB, 2014.

SARNO, F.; CLARO, R. M.; LEVY, R. B.; BANDONI, D. H.; MONTEIRO, C. A. Estimated sodium intake for the Brazilian population, 20082009. Revista de Saúde Pública, v. 47, n. 3, p. 1-7, 2013.

SILVA, A. S.; COUTINHO, V. F.; AZEVEDO, C. H. Análise do teor de sódio em molhos de tomate industrializados: um alerta para hipertensão. Revista Saber Científico, v. 4, n. 1, p. 38-46, 2015.

SOCIEDADE BRASILEIRA DE HIPERTENSÃO (SBH). Benefícios da atividade física. 2011. Disponível em: <http:www.sbh.org.br>. Acesso em: 16 abr. 2016. 
TOBIN, B. D.; O'SULLIVAN, M. G.; HAMILL, R. M.; KERRY, J. P. Effect of varying salt and fat levels on the sensory quality of beef patties. Meat Science, v. 91, n. 4, p. 460-465, ago. 2012.

UNITED STATES DEPARTMENT OF AGRICULTURE (USDA). Standard Release 25 - Tabela de composição química dos alimentos. 2012. Disponível em: <http://www.dis.epm.br/ servicos/nutri/public/ alimento/nutriente/ndbno/21228>. Acesso em: 20 jan. 2016.

VENTURA, R. Mudanças no perfil do consumo no Brasil: principais tendências nos próximos 20 anos. Rio de Janeiro: Macroplan Prospectiva, Estratégia e Gestão, ago. 2010.

VIGILÂNCIA DE FATORES DE RISCO E PROTEÇÃO PARA DOENÇAS CRÔNICAS POR INQUÉRITO TELEFÔNICO (VIGITEL). Obesidade estabiliza no Brasil, mas excesso de peso aumenta. 2014. Disponível em: <http://portalsaude.saude.gov.br/index.php/o-ministerio/principal/ secretarias/svs/noticias-svs/17455-obesidade-estabiliza-no-brasilmas-excesso-de-peso-aumenta>. Acesso em: 26 maio 2016.

WORLD HEALTH ORGANIZATION (WHO). Diet, nutrition and prevention of chronic diseases. Report FAO/WHO Expert Consultation. WHO Technical Report Series, Geneva, n. 916, 2003.

. Who issues new guidance on dietary salt and potassium 2013. Disponível em: <http://www.who.int/mediacentre/news/notes/2013/ salt_potassium_20130131/en/>_. Acesso em: 13 maio 2016.

YOKOO, E. M.; MASSARANI, F. A.; SOUZA, B. N.; MURARO, A. P.; CUNHA, D. B. Correlação de padrão alimentar do tipo "fast food" entre pais e filhos no Brasil. In: CONGRESSO NACIONAL DA SBAN, 12., 13 a 16 de agosto de 2013. Anais... Foz do Iguaçu, PR, 2013. 